\title{
Study on Backward Scattering Characteristics of Submicron Particles
}

\author{
Keliang Hou*, Chunwei Wang, Xiang Liu \\ College of Science, University of Shanghai for Science and Technology, Shanghai, China \\ Email: *382954886@qq.com
}

How to cite this paper: Hou, K.L., Wang, C.W. and Liu, X. (2020) Study on Backward Scattering Characteristics of Submicron Particles. Optics and Photonics Journal, 10, 79-87.

https://doi.org/10.4236/opj.2020.105007

Received: April 14, 2020

Accepted: April 27, 2020

Published: April 30, 2020

Copyright $\odot 2020$ by author(s) and Scientific Research Publishing Inc. This work is licensed under the Creative Commons Attribution International License (CC BY 4.0).

http://creativecommons.org/licenses/by/4.0/

\section{(c) (i) Open Access}

\begin{abstract}
In production and scientific research, the research on the characteristics of submicron particles has attracted extensive attention, which is of great significance to the development of industrial production, medical and health inspection and other fields. In this paper, the characteristics of submicron particles are studied based on backlight scattering. By collecting the real-time waveform of the backscattering signal of particles with different particle sizes at different concentrations, the corresponding relationship between the particle concentration and the number of pulses was obtained. It is found that the peak value of the backscattered light energy has a good linear relationship with the particle size. The analysis of the signal distribution law of standard particle swarm with different particle size parameters found that it conformed to the lognormal distribution form.
\end{abstract}

\section{Keywords}

Backward Scattering, Submicron Particles, Lognormal Distribution

\section{Introduction}

Submicron particles are widely present in production and daily life [1] [2] [3] [4] [5]. For example, the line width and pitch of large-scale integrated circuits are very small, which puts forward higher requirements on the production technology of the circuit and its manufacturing environment. Not only should submicron or even nano lines be etched, but circuit defects must be controlled to a certain range in order to ensure the function and yield of the chip. However, the particulate matter generated in the production process and the particulate matter in the production environment will make the integrated circuit lose its proper function and become a waste product. Therefore, we need real-time detection of submicron particles in the environment. In this paper, based on the genera- 
lized Mie scattering theory [6] [7], the relationship between backscattered light energy and particle size is obtained through numerical simulation calculations. The relationship between the backlight scattering energy and particle size and particle concentration was studied based on the backlight scattering particle testing technology.

\section{Backscatter Calculation Principle}

The experimental system for measuring the backscattering characteristics of particles is shown in Figure 1. The laser light emitted by the laser is irradiated by the Gaussian beam condensed by the transflective mirror and lens combination into the flowing sample cell. The backscattered light of the particles passes through the same lens combination and transflective mirror to be accepted and transformed into electrical signals by the detector. Finally, the electrical signals are sent to the computer for processing.

Figure 2 is the calculated coordinates of the spatial distribution of scattered light of particles based on the generalized Mie theory [8] [9] [10] (abbreviated as GLMT), where $O_{G} \mu v \omega$ is the beam coordinate system, the beam propagates along the $w$ direction, and the gaussian beam waist center is $O_{G}$. The coordinate

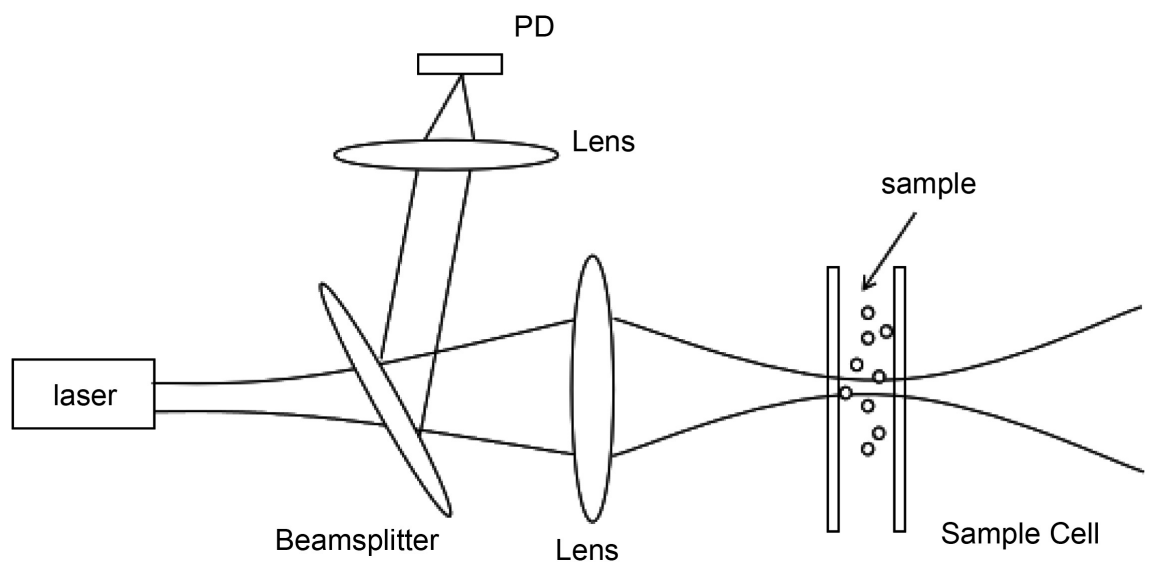

Figure 1. Schematic diagram of backward light scattering particles.

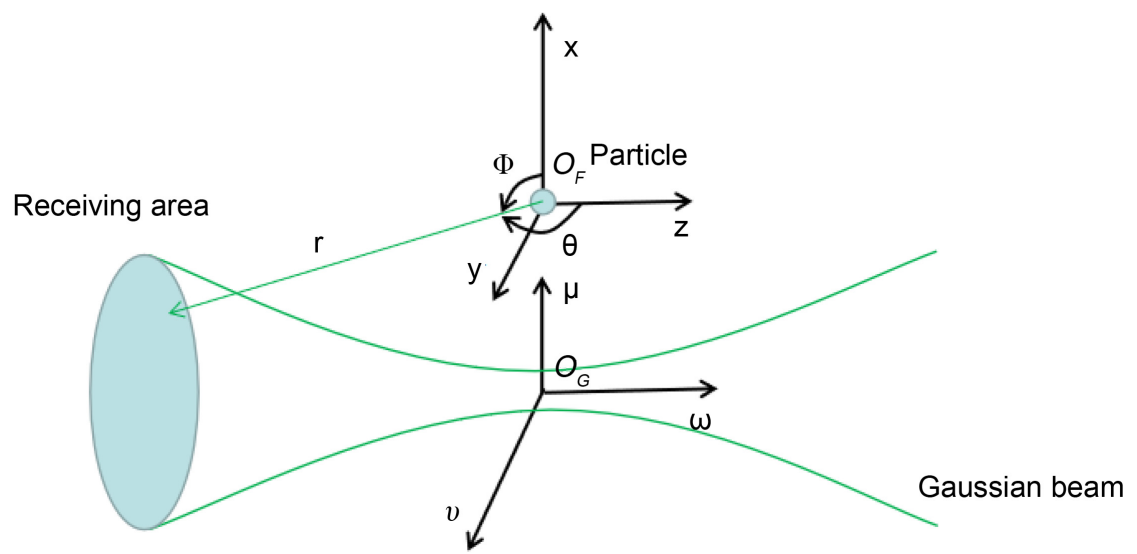

Figure 2. Model for calculating the spatial distribution of scattered light from particles. 
system $O_{p} x y z$ is the particle coordinate system, the origin of the coordinate system is at the particle center, the $z$-axis is parallel to the $w$ axis.

According to the calculation model shown in Figure 2, in the actual calculation, it is necessary to set a position of the particles in the beam coordinate system; set the integration range $\alpha \in[0,2 \pi]$ and $\rho \in\left[0, r_{\max }\right]$ of the detection point. Calculate the scattered light energy within this integration range, namely:

$$
E_{\text {sca }}=\int_{\alpha=0}^{2 \pi} \int_{\rho=0}^{\rho_{\max }} I_{\text {sca }}(\theta, \phi) \rho \mathrm{d} \rho \mathrm{d} \alpha .
$$

among them

$$
I_{\text {sca }}(\theta, \phi)=\frac{\lambda^{2}}{4 \pi^{2} r^{2}}\left[i_{1}(\theta, \phi)+i_{2}(\theta, \phi)\right] .
$$

In the above formula, $i_{1}(\theta, \phi)$ and $i_{2}(\theta, \phi)$ are scattering intensity functions, which can be calculated by GLMT.

\section{Relationship between Particle Size and Backscattered Light Energy}

The backscattering characteristics of the particles are closely related to the particle size. With different particle sizes, the backscattered light energy is significantly different. Based on the principle of backscattering of particles, a program written by Monte Carlo algorithm is used to calculate the backscattered light energy of the particles, and the corresponding relationship between the scattered light energy of the particles and the particle size is obtained by changing the parameters. Set the beam waist radius of the Gaussian beam to be $\omega=5.0$, the wavelength to be $\lambda=0.6328$, and the refractive index is 1.57 , change the particle size. From the simulation calculations, it can be known that the backscattered light energy has a linear relationship of linear micro-oscillation on the logarithmic coordinate as the particle size changes. The line relationship is shown in Figure 3.

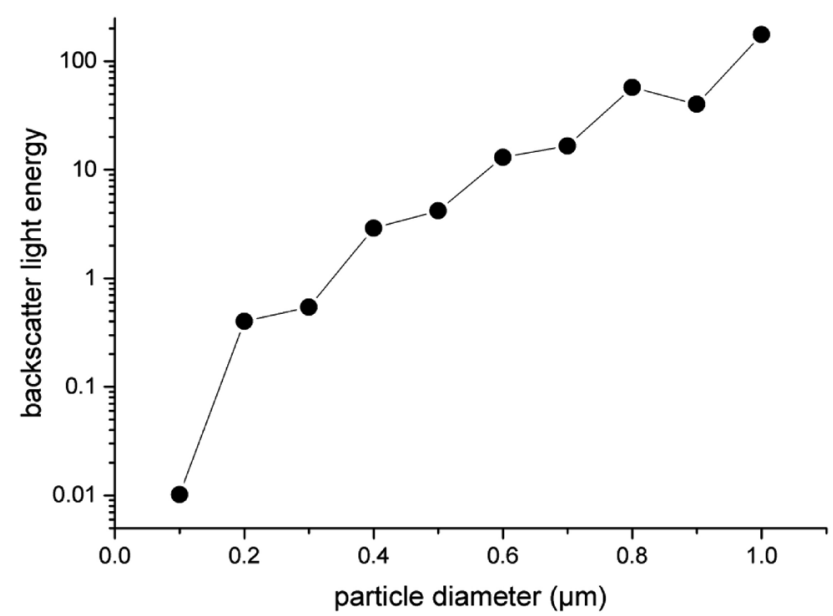

Figure 3. Relationship between particle backscattered light energy and particle size. 


\section{Experimental Research}

The experimental device is built according to the experimental principle of Figure 1, in which the amplifier circuit adopts the two-stage amplification mode of the LT1793 op-amp in series with LT1012. By adjusting the resistance ratio of the chip input terminal to control the amplification factor, the input optical signal can be converted into a current signal and then into a voltage signal. The space where the liquid flows inside the sample cell is a rectangular parallelepiped, in which the height of the rectangular parallelepiped is $150 \mathrm{~mm}$, and the cross-section is a square with a side length of $10 \mathrm{~mm}$. The circulating pump system adopts BT800 type peristaltic pump, and the circulating speed is adjustable from $500 \mathrm{rev} / \mathrm{min}$ to $2000 \mathrm{rev} / \mathrm{min}$. The signal acquisition software is the top view 2000 oscilloscope simulation system of Sichuan Tuopu Measurement \& Control Technology Co., Ltd. It adopts the internal trigger mode and sets the trigger voltage to $0 \mathrm{~V}-10 \mathrm{~V}$.

The standard polystyrene particles with radii of $0.1 \mu \mathrm{m}, 0.2 \mu \mathrm{m}, 0.3 \mu \mathrm{m}, 0.5$ $\mu \mathrm{m}$ were measured by the above experimental device, and the pulse waveform signal was obtained. Before putting the particles to be tested, start the peristaltic pump, adjust the photoelectric detector, and reduce the noise.

Figure 4 shows the pulse waves of these four particles measured in the laboratory and Gaussian fitting the waveform with origin. Figure 5 shows the relationship between the peak values of these four-particle pulse waveforms and particle size, and it is found that the linear relationship is basically consistent with Figure 3.

\section{Relationship between Backscatter Signal and Particle Concentration}

Under the same experimental conditions, particle samples were gradually added to the cyclic sampling system. Figure 6 shows the experimental results of dropping 2 drops, 3 drops and 4 drops of particles with a particle size of 0.3. From the figure, we can see that the number of particle backscattering response signals detected increases with increasing concentration during the same period. The higher the concentration in the same period of time, the more particles pass through the measurement area.

\section{Statistics of Particle Light Scattering Pulse Signal Count Distribution}

In Figure 4, the scattered light energy of different particles is the maximum value selected from a large amount of data obtained in the experiment, but this does not guarantee that the particles pass through the center of the beam waist, so a large amount of data is required to count the relationship between the light scattering pulse signal and the particles. During the experimental measurement, the particles in the liquid passing through the Gaussian beam waist area are random. Therefore, the amplitude of the backscattered pulse signal generated by 

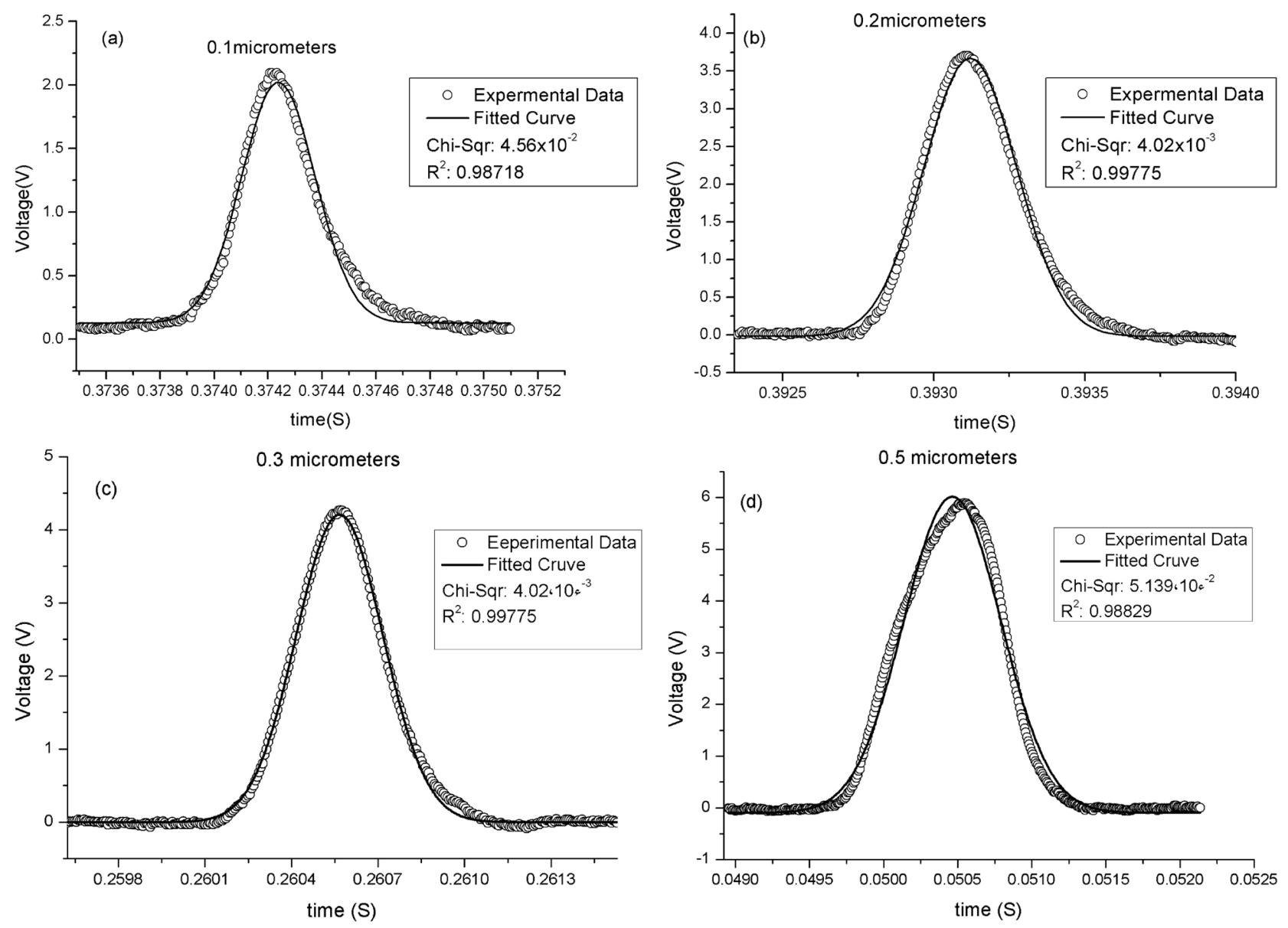

Figure 4. Response waveforms and Gaussian fit curves of polystyrene standard particles with different particle sizes. (a) the particle waveforms of $0.1 \mu \mathrm{m}$; (b) the particle waveforms of $0.2 \mu \mathrm{m}$; (c) the particle waveforms of $0.3 \mu \mathrm{m}$; (d) the particle waveforms of $0.5 \mu \mathrm{m}$.

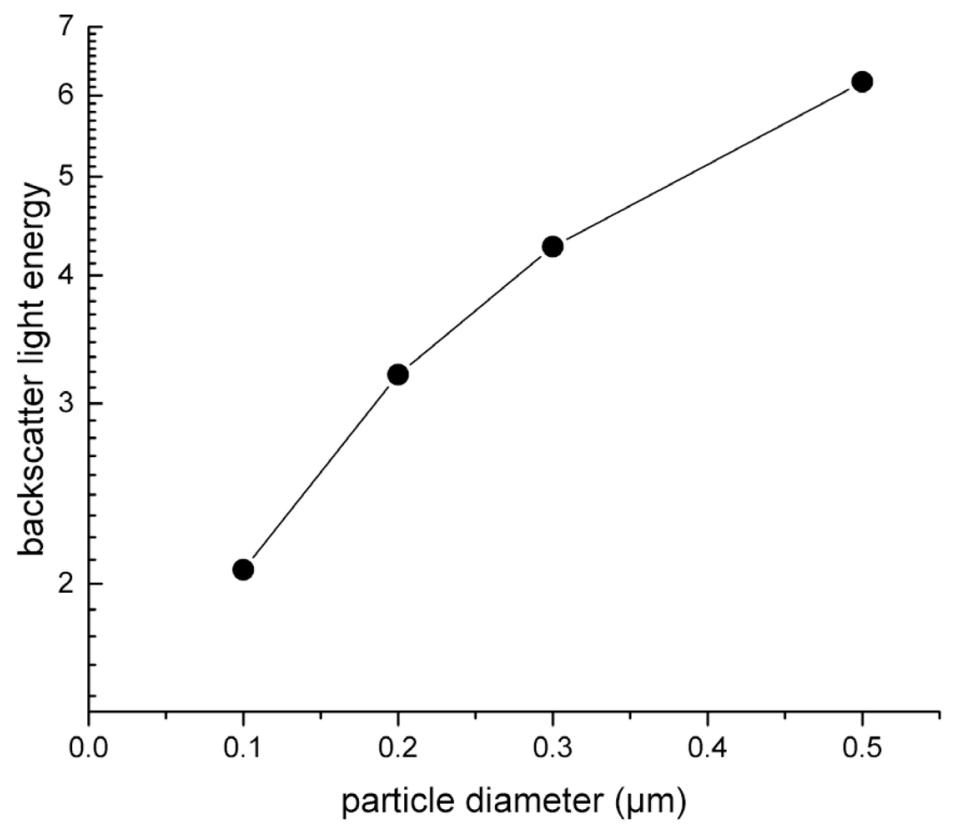

Figure 5. Relationship between particle size and its peak. 

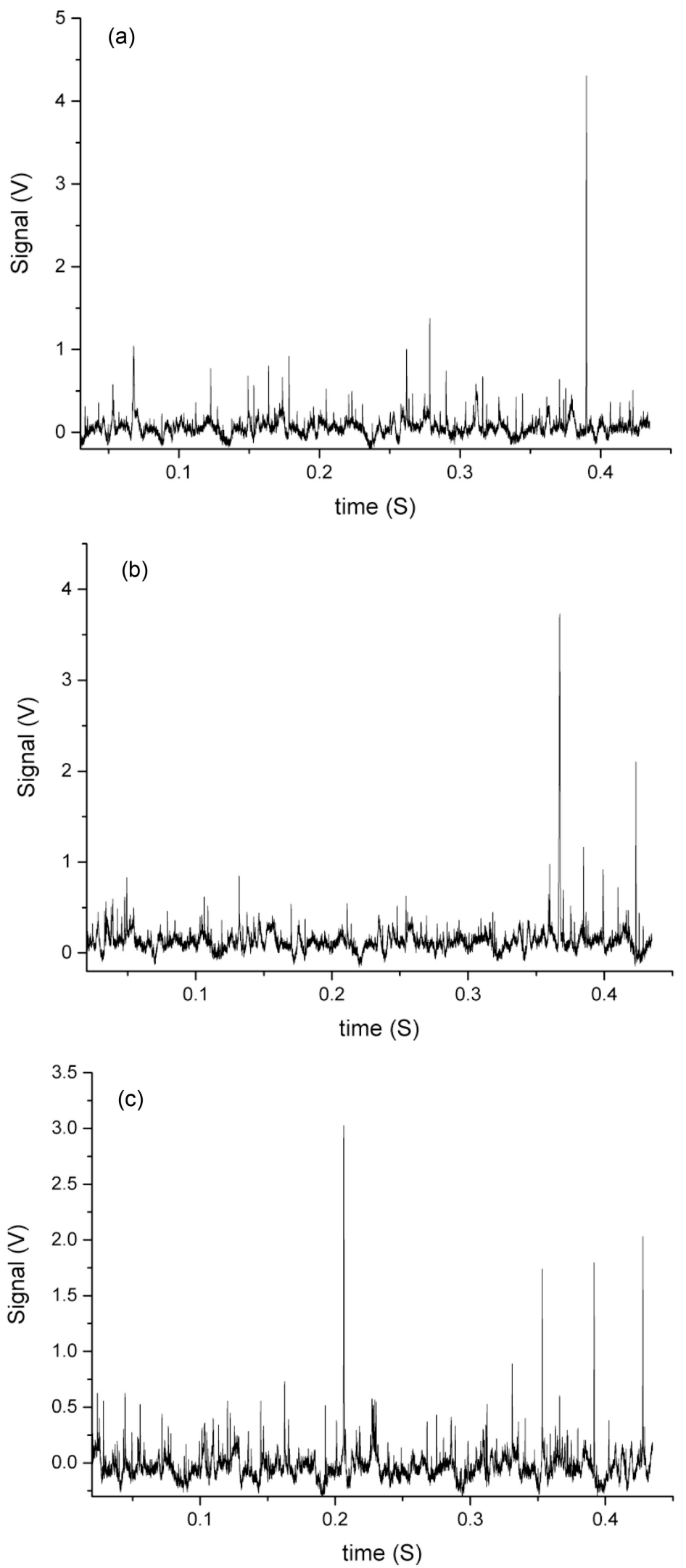

Figure 6. Scattered signals at different concentrations. (a) 2 drops of particles; (b) 3 drops of particles; (c) 3 drops of particles. 
the particles is also random, and the amplitude of these pulse signals contains the inherent characteristics of the interaction between the particles and the beam (such as particle size, particle refractive index, etc.).

In this paper, we try to fit the logarithmic normal distribution to the amplitude of the experimental data, equation [11] is as follows:

$$
P\left(V-V_{m}\right)=\frac{1}{\sqrt{2 \pi} \sigma_{v}\left(V-V_{m}\right)} \exp \left[-\frac{\left(\ln \left(V-V_{m}\right)-\mu_{v}\right)^{2}}{2 \sigma_{v}^{2}}\right], V \in\left(V_{m}-V_{M}\right)
$$

where $V_{m}$ represents the classification interval of voltage, $V$ represents the voltage value, and $P\left(V-V_{m}\right)$ represents the probability value between $V$ and $V+V_{m}$.

Under the same experimental device conditions, random measurement is performed on the standard particles with particle sizes of $0.2 \mu \mathrm{m}, 0.3 \mu \mathrm{m}$, and 0.5 $\mu \mathrm{m}$ to obtain 1000 pieces of data respectively, and the peak value of these 1000 pieces of data was counted according to formula (3) using Python language. The data results are shown in Figure 7, where the $\mathrm{x}$-axis represents voltage and the $y$-axis represents probability. It can be seen from the figure that the distribution of the pulse signal does not satisfy the center symmetry, and the voltage corresponding to the peak of the amplitude distribution increases as the particle size increases.

\section{Conclusions}

This article introduces a submicron particle test method for detecting backscattered light signals. In the detection device of this method, the incident Gaussian

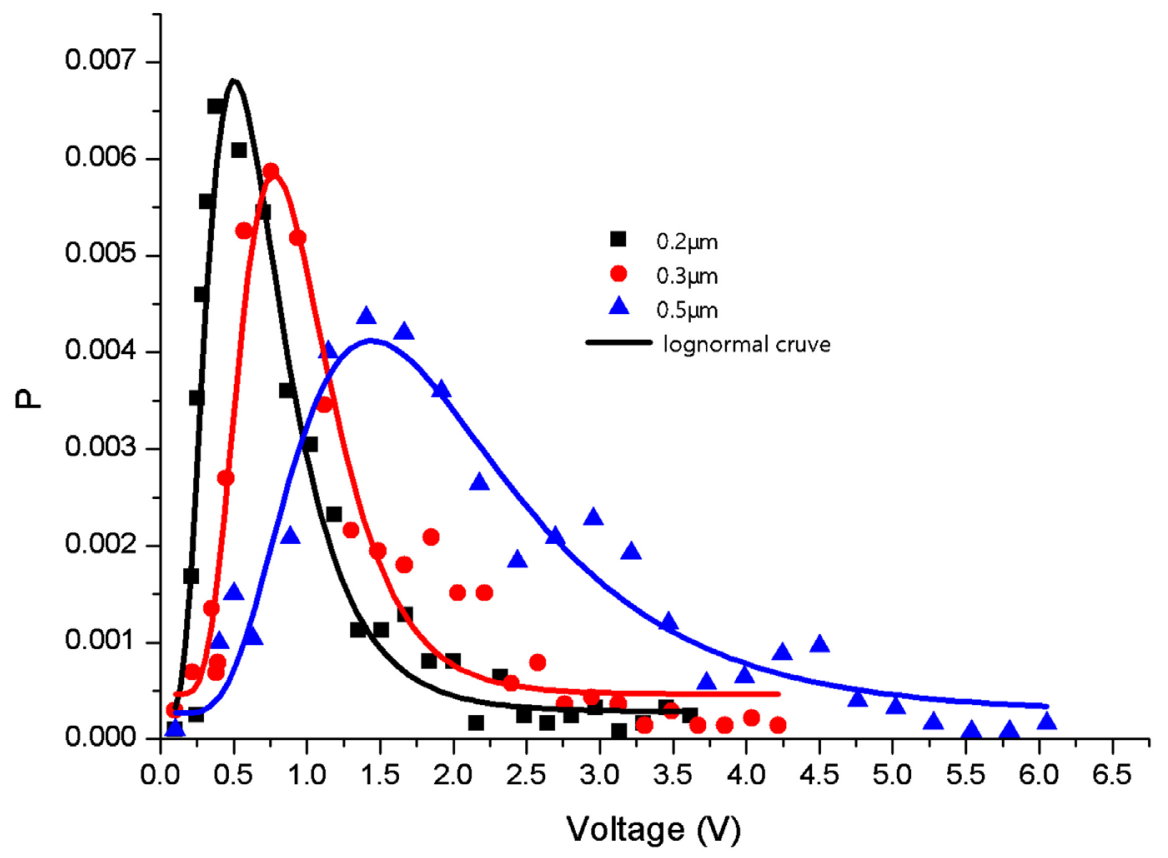

Figure 7. Experimental (point) and fitting (solid line) results of the amplitude distribution of the laser scattering pulse signal. 
beam and the scattered beam pass through the same lens, which makes the measurement area independent of the optical device, thereby enhancing the shockproof performance of the measurement device, which is convenient for practical applications.

Through theoretical analysis and experimental research on the backscattering method, the article obtains the variation law between the particle size and particle concentration and the backscattered light energy. When the number of samples is collected enough, the peak distribution of the pulse waveform is analyzed and it is found that the statistical amplitude distribution of the particles agrees well with the lognormal distribution. The experimental measurement shows that the backscattered light energy distribution of the particles has a linear relationship with the particle size and is consistent with the theoretical analysis results, and the number of particle backscattering response signals in the same time period increases with increasing concentration.

\section{Conflicts of Interest}

The authors declare no conflicts of interest regarding the publication of this paper.

\section{References}

[1] Savkin, K.P., Bugaev, A.S., Gushenets, V.I., Nikolaev, A.G., Ivanov, Y.F., Oks, E.M., Frolova, V.P., Shandrikov, M.V. and Yushkov, G.Y. (2020) Generation of Micron and Submicron Particles in Atmospheric Pressure Discharge in Argon Flow with Magnesium, Zinc, and Boron Carbide Electrodes. Surface and Coatings Technology, 389, Article ID: 125578. https://doi.org/10.1016/j.surfcoat.2020.125578

[2] Cao, L.M., Zhu, Q., Huang, X.F., Deng, J.J., Chen, J.S., Hong, Y.W., Xu, L.L. and He, L.Y. (2016) Chemical Characterization and Source Apportionment of Atmospheric Submicron Particles on the Western Coast of Taiwan Strait, China. Journal of Environmental Sciences, 52, 293-304. https://doi.org/10.1016/j.jes.2016.09.018

[3] Toraman, O.Y. (2015) Production of Submicron Particles by Mechanical Treatment: Width of Particle Size Distribution and Fineness of Product. Particulate Science and Technology, 33, 666-670. https://doi.org/10.1080/02726351.2015.1020180

[4] Tan, Z.C., Givehchi, R. and Saprykina, A. (2015) Submicron Particle Sizing by Aerodynamic Dynamic Focusing and Electrical Charge Measurement. Particuology, 18, 105-111. https://doi.org/10.1016/j.partic.2014.01.002

[5] Mora-Huertas, C.E., Fessi, H. and Elaissari, A. (2011) Influence of Process and Formulation Parameters on the Formation of Submicron Particles by Solvent Displacement and Emulsification-Diffusion Methods: Critical Comparison. Advances in Colloid and Interface Science, 163, 90-122.

https://doi.org/10.1016/j.cis.2011.02.005

[6] Bohren, C.F. and Huffman, D.R. (1998) Absorption and Scattering of Light by Small Particles. Wiley-VCH Verlag GmbH, Weinheim, Germany. https://doi.org/10.1002/9783527618156

[7] Van de Hulst, H.C. (1957) Light Scattering by Small Particles. John Wiley and Sons, New York. https://doi.org/10.1063/1.3060205 
[8] Gouesbet, G. and Gréhan, G. (2011) Generalized Lorenz-Mie Theories. Springer, Berlin. https://doi.org/10.1007/978-3-642-17194-9

[9] Gouesbet, G., Maheu, B. and Gréhan, G. (1988) Light Scattering from a Sphere Arbitrarily Located in a Gaussian Beam, Using a Bromwich Formulation. Journal of the Optical Society of America A, 5, 1427-1443.

https://doi.org/10.1364/JOSAA.5.001427

[10] Maheu, B., Gouesbet, G. and Gréhan, G. (1988) A Concise Presentation of the Generalized Lorenz-Mie Theory for Arbitrary Location of the Scatterer in an Arbitrary Incident Profile. Journal of Optics, 19, 59-67. https://doi.org/10.1088/0150-536X/19/2/002

[11] Yan, Z.G., Sun, W.P., Yan, K.D., et al. (2014) Random Laser Scattering Pulse Signal Analysis in Laser Particle Counter with Lognormal Distribution. Proceedings of Real-Time Photonic Measurements, Data Management, and Processing, Beijing, Vol. 9279. https://doi.org/10.1117/12.2071057 